\title{
A trial comparing continuous positive airway pressure (CPAP) devices in preterm infants
}

Carl H. Backes ${ }^{1,2,3} \cdot$ Jennifer N. Cooper ${ }^{2,4,5} \cdot$ Jennifer L. Notestine ${ }^{1} \cdot$ Crystal M. Alfred $^{2} \cdot$ Molly K. Ball $^{2}$. Brian K. Rivera ${ }^{1} \cdot$ Jane M. Lamp ${ }^{1} \cdot$ Laura Marzec $^{1} \cdot$ Michael R. Stenger $^{2} \cdot$ Mohannad Moallem $^{2} \cdot$ Randy R. Miller $^{2,6}$.

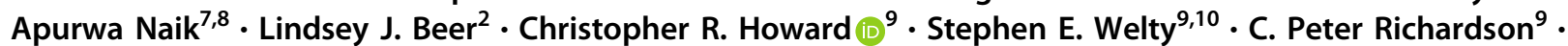
Noah H. Hillman ${ }^{11}$ • John A. F. Zupancic $\mathbb{D}^{12,13} \cdot$ Larissa I. Stanberry ${ }^{14} \cdot$ Thomas N. Hansen $^{9} \cdot$ Charles V. Smith ${ }^{9}$

Received: 24 February 2020 / Revised: 6 April 2020 / Accepted: 7 May 2020 / Published online: 20 May 2020

(c) The Author(s) 2020. This article is published with open access

\begin{abstract}
Objective To test the hypothesis that infants born $<30$ weeks' gestation supported by Seattle-PAP will have lower rates of continuous positive airway pressure (CPAP) failure than infants supported with conventional, Fisher\&Paykel-CPAP (FPCPAP).

Study design Randomized trial (3/2017-01/2019) at 5 NICUs. The primary outcome was CPAP failure; subgroup analyses (gestational age, receipt antenatal corticosteroids) were performed.

Results A total of 232 infants were randomized. Infants in the Seattle-PAP and FP-CPAP groups had mean gestational ages of 27.0 and 27.2 weeks, respectively. We observed no differences in rates of treatment failure between Seattle-PAP (40/112, $35.7 \%)$ and FP-CPAP (38/120, 31.7\%; risk difference, $4.1 \%$; 95\% CI, $-8.1-16.2 ; P=0.51)$. Subgroup analysis indicated no differences in rates of CPAP failure. We observed no differences between the two groups in frequencies of adverse events or duration of respiratory support.

Conclusions Among infants born $<30$ weeks' gestation, rates of CPAP failure did not differ between Seattle-PAP and FP-CPAP.
\end{abstract}

Supplementary information The online version of this article (https:// doi.org/10.1038/s41372-020-0690-5) contains supplementary material, which is available to authorized users.

Carl H. Backes

Carl.backesjr@nationwidechildrens.org

1 Center for Perinatal Research, The Abigail Wexner Research Institute at Nationwide Children's Hospital, Columbus, OH, USA

2 Department of Pediatrics, The Ohio State University Wexner Medical Center, Columbus, OH, USA

3 The Heart Center, Nationwide Children's Hospital, Columbus, $\mathrm{OH}$, USA

4 Center for Surgical Outcomes, The Abigail Wexner Research Institute at Nationwide Children's Hospital, Columbus, OH, USA

5 Division of Epidemiology, The Ohio State University College of Public Health, Columbus, OH, USA

6 Department of Pediatrics, Mt. Carmel St. Ann's Hospital, Westerville, OH, USA

\section{Introduction}

Respiratory distress in the perinatal period is common among preterm infants, particularly among those born at $<30$ weeks of gestation [1]. The American Academy of Pediatrics has endorsed the use of continuous positive

7 OhioHealth Grant Medical Center, Columbus, OH, USA

8 Central Ohio Newborn Medicine, Columbus, OH, USA

9 Seattle Children's Research Institute, Seattle Children's Hospital, Seattle, WA, USA

10 Seattle Children's Neonatology Program, CHI Franciscan Health, Tacoma, WA, USA

11 SSM Health, Cardinal Glennon Children's Hospital, St. Louis University, St. Louis, MO, USA

12 Division of Newborn Medicine, Harvard Medical School, Boston, MA, USA

13 Department of Neonatology, Beth Israel Deaconess Medical Center, Boston, MA, USA

14 Minneapolis Heart Institute Foundation, Minneapolis, MN, USA 
airway pressure (CPAP) among preterm infants with respiratory distress [2], based on lower rates of the combined outcome of bronchopulmonary dysplasia (BPD) or death [3]. CPAP failure, defined as the need for tracheal intubation and mechanical ventilation, is common among preterm infants $<30$ weeks of gestation, with failure rates approaching $45-50 \%$ in large clinical trials [4-7]. In developing countries and resource-limited facilities in which intubation and mechanical ventilation is not available, CPAP failure is associated with greater mortality [8]. Thus, preventing CPAP failure remains a high priority among health care providers caring for preterm infants [9]. These observations led to the design and development of a novel, low-cost, CPAP system-Seattle-PAP.

In traditional bubble CPAP systems, the expiratory limb of the circuit is oriented vertically into the water seal of the bubbler apparatus $\left(0^{\circ}\right)$. In contrast, the expiratory limb of the circuit in Seattle-PAP is maintained at $135^{\circ}$ (Fig. 1), which leads to fluctuations in airway pressure that are notably different than with traditional bubble CPAP systems [8, 10-12]. In preclinical studies, Seattle-PAP resulted in higher arterial oxygen pressure $\left(\mathrm{PaO}_{2}\right)$ levels and markedly lower $(50 \%)$ work of breathing, than conventional CPAP systems [11]. In addition, in a small, single-center study of preterm infants (average gestational age of 29 weeks), breathing effort was lower with Seattle-PAP than with conventional Fisher \& Paykel bubble CPAP (FP-CPAP), with no indications of increased rates of adverse events; however, that study was not designed to test differences with Seattle-PAP on clinical outcomes [12]. Despite the strength of the pre-clinical and early clinical studies, few centers have extensive experience with Seattle-PAP. We therefore conducted a multicenter, randomized controlled trial (RCT) to test the hypothesis that premature infants ( $<30$ weeks of gestation) supported by Seattle-PAP would exhibit lower rates of CPAP failure than would neonates supported with FP-CPAP.

\section{Patients and methods}

\section{Participating institutions, study design, and oversight}

The Nationwide Children's Hospital (NCH) IRB approved the trial (\#16-00678); review was ceded to the NCH IRB by Riverside Methodist Hospital, Grant Medical Center, and The Ohio State University Wexner Medical Center. The IRB at Mt. Carmel St. Ann's Hospital granted separate institutional approval (\#170817-2). The facilities routinely care for premature infants with respiratory distress, using FP-CPAP as the primary noninvasive respiratory support modality. Participating sites did not use Seattle-PAP prior

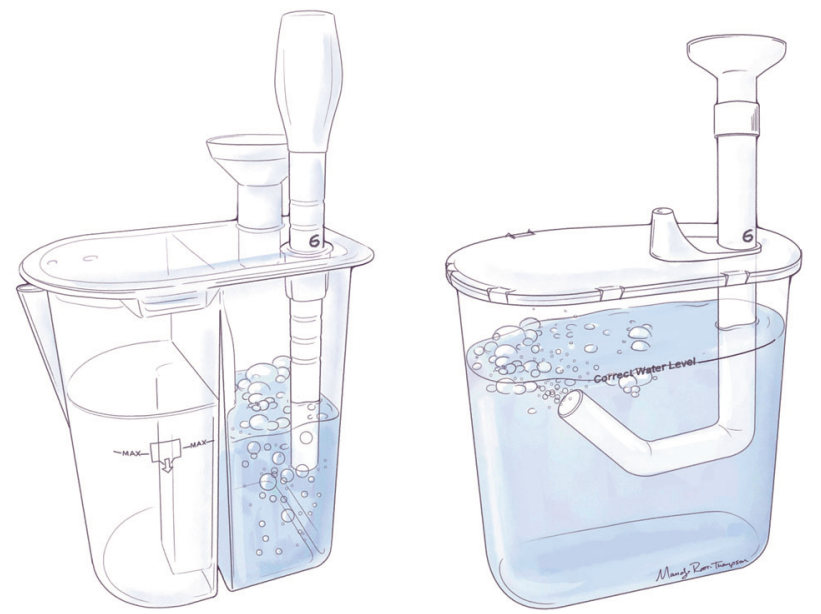

Fig. 1 Illustration of Fisher \& Paykel (left) and Seattle-PAP (right) bubble CPAP generators. The expiratory limb of the circuit is oriented vertically into the water seal of the bubbler apparatus $\left(0^{\circ}\right)$ in Fisher \& Paykel, while the expiratory limb of the circuit in Seattle$\mathrm{PAP}$ is maintained at $135^{\circ}$.

to study commencement. The trial was registered with ClinicalTrials.gov (NCT03085329). An external safety and efficacy monitoring board (DSMB) conducted regular reviews of patient safety (treatment $\mathrm{A}$ vs. treatment $\mathrm{B}$ ) using compiled data summaries. Details regarding the trial study design and protocol have been published previously [13].

\section{Eligibility and recruitment}

Infants were eligible for inclusion if delivered at gestational ages between $22^{0 / 7}-29^{6 / 7}$ weeks and if they were candidates for noninvasive respiratory support, either as: 1) initial support between birth and $72 \mathrm{~h}$ of age; or 2) following the initial extubation and withdrawal of mechanical support during the first $72 \mathrm{~h}$ of life. Infants were excluded if their postnatal ages were $>72 \mathrm{~h}$ at the time of extubation, they had a known congenital anomaly (including any airway abnormality [e.g., Pierre-Robin sequence and cleft lip and palate]) that might adversely affect breathing, had a known genetic anomaly (e.g., Trisomy 21), or if maximal intensive care was not being provided. Infants were recruited at a large US pediatric academic medical center with five participating neonatal intensive care units (NICUs) that share guidelines in the care and management of preterm infants. Infants transported from outside institutions, if meeting eligibility criteria, were also approached. Study team members obtained written, informed consent from parents or guardians of eligible infants; antepartum consent was sought, when possible. If antepartum consent was not obtained, parents/guardians of eligible infants were approached as soon as possible following delivery, up to $72 \mathrm{~h}$ ' postnatal age. 


\section{Randomization and blinding}

Upon receipt of consent and verification of eligibility, treatment assignments were performed with the use of a secure study website. Participants were assigned randomly to treatment with either Seattle-PAP or FP-CPAP. Due to the nature of the intervention, blinding as to the assigned treatment arms was not possible with respect to the treatment teams. Blinding was achieved for data analysis purposes by assigning generic identifiers among trial participants prior to data transfer. We did not stratify by site, as treatment of infants and additional aspects of care were based on shared guidelines among the five participating NICUs [13, 14].

\section{Treatment strategies}

\section{Delivery room management}

Among mother-infant dyads consented antenatally, the assigned CPAP device (Seattle-PAP or FP-CPAP) was initially offered to infants as the primary respiratory modality; however, INSURE (INtubate, SURfactant, Extubate) was permitted [9]. Delivery room management (e.g., criteria for intubation), followed international guidelines, including those of the Neonatal Resuscitation Program [15, 16]. For mother-infant dyads that consented postnatally, randomization to the assigned CPAP device was performed at the time of the first decision to use noninvasive support.

\section{Guidelines for extubation to CPAP from mechanical ventilation}

Weaning from mechanical ventilation was based on established guidelines across participating NICUs [13, 14]. Extubation was recommended within $24 \mathrm{~h}$ of meeting all the following criteria: fraction of inspired oxygen $\left(\mathrm{FiO}_{2}\right)$ of 0.30 or less with oxygen saturation as measured by pulse oximetry $\left(\mathrm{SpO}_{2}\right)$ of $92 \%$ or greater, mean airway pressure of $8 \mathrm{~cm} \mathrm{H}_{2} \mathrm{O}$ or less with hemodynamic stability, and receiving caffeine (either a loading dose of $20 \mathrm{mg}$ per kilogram body weight or a maintenance dose of $5 \mathrm{mg}$ per kilogram) [17]. While the guidelines emphasize administration of exogenous surfactant therapy for infants on mechanical ventilation with $\mathrm{FiO}_{2}>0.40$, use was at the discretion of the attending provider.

\section{Guidelines for intubation}

Infants who could not be maintained with their assigned CPAP device were intubated and ventilated; the originally assigned intervention was resumed after extubation. Consistent with previous studies, intubation was recommended if: 1) more than two episodes of apnea requiring bag-mask ventilation were encountered in a $24 \mathrm{~h}$ period or more than six episodes requiring any intervention within a $6 \mathrm{~h}$ period; 2) $\mathrm{FiO}_{2}$ of 0.50 or greater to maintain $\mathrm{SpO}_{2}$ of $88 \%$; 3) cardiovascular instability; 4) as recommended by treating health care provider $[17,18]$. Among infants with apnea, administration of caffeine was stressed in the guidelines, but at the discretion of the attending provider. All participating NICUs placed an emphasis on minimizing laboratory studies; thus, arterial or venous blood gases $(\mathrm{pH}$, carbon dioxide) were not included in the recommendations for reintubation [14]. Adherence to treatment guidelines were monitored and reported [13].

\section{Guidelines for weaning from CPAP}

Based on expert opinion, for infants requiring $\mathrm{FiO}_{2}>0.21$, weaning from the assigned CPAP device to any nasal cannula, including high-flow nasal cannula (HFNC), was not endorsed prior to 32 weeks' postmenstrual age [19]. Failure to maintain target oxygen saturations on room air without respiratory support prompted return of the infant to the assigned CPAP device.

\section{Respiratory care for infants on CPAP}

In the absence of clear data to guide the practice of evidence-based medicine, and reflecting current practice patterns, the use of chin-straps and pacifiers, and body positioning (prone, lateral), were not specified [9]. To avoid erosive damage and minimize the risk of subsequent nasal deformities, nasal prongs were chosen that: 1) fit securely in the infant's nares; 2) avoided pinching the septum or blanching of the nares. CPAP was delivered though either short bi-nasal prongs or a nasal mask [20]. To decrease the risk of nasal injury, prongs, and masks were alternated every 3-6 h [21]. The frequency of gentle suctioning of the nasal cavities, oropharynx, and stomach was recommended every $3-4 \mathrm{~h}$; however, guidelines stressed that suctioning needs should be dictated by clinical assessment (e.g., increased oxygen requirement, increased work of breathing). In most cases, a 6 French (Fr) catheter was used for suctioning, with a continuous suction pressure of $-80 \mathrm{mmHg}$. Additional details on treatment guidelines for the present study have been previously published [13].

\section{Study outcomes}

The primary outcome was CPAP treatment failure, defined by the following: 1) tracheal intubation within $72 \mathrm{~h}$ for surfactant administration after initiation of bubble CPAP and then not extubated by $72 \mathrm{~h} ; 2$ ) tracheal intubation or 
support with biphasic CPAP (synchronized nasal intermittent positive pressure ventilation, SiPAP) after $72 \mathrm{~h}$ postdelivery and up to 32 weeks gestational age; 3 ) inability to sustain $\mathrm{SpO}_{2}$ of at least $90 \%$, despite noninvasive respiratory support of $8 \mathrm{~cm} \mathrm{H}_{2} \mathrm{O}$ bubble CPAP, and $\mathrm{FiO}_{2}>0.40$ for more than $1 \mathrm{~h}$. Consistent with previous studies [18], $72 \mathrm{~h}$ post-delivery was chosen to provide a window for surfactant administration. As adjudicated by an independent investigator, tracheal intubations for surfactant delivery (within $72 \mathrm{~h}$ post-delivery) and non-respiratory issues (e.g., surgery for retinopathy of prematurity) were not considered treatment failures.

Pre-specified secondary outcomes included reasons for treatment failure, time (hours) to treatment failure after randomization, days of mechanical ventilation (synchronized intermittent mandatory ventilation [SIMV] or highfrequency oscillatory ventilation [HFOV]) after trial entry, days of any positive pressure (SIMV, HFOV, SiPAP, CPAP, and HFNC), days of oxygen therapy after trial entry, days on room air after trial entry, days to achieve full enteral feeds $(>130 \mathrm{~mL} / \mathrm{kg} / \mathrm{day})$ after trial entry, days to achieve full-suck feeding ( $>130 \mathrm{ml} / \mathrm{kg} /$ day) after trial entry, and weight at discharge. Differences in CPAP failure rates among infants who had antenatal consent and were started on CPAP at birth were examined. The complete list of prespecified secondary outcomes is provided in the published study protocol [13]. Serious adverse events were defined in the previously-published study protocol [13]. These events were reported to the data safety and monitoring board (DSMB) as they occurred. Data were collected until death or discharge home.

\section{Statistical analysis}

Based on data from previous clinical trials of preterm infants using CPAP, we estimated a treatment failure rate of $50 \%$ in the control arm (FP-CPAP) [4-6]. From earlier studies, we estimated that treatment failure rates of SeattlePAP to be $30 \%[8,12]$. As multiples were to be randomized as a set to the same study arm, the sample size estimate was inflated by 1.12 , to allow for the effect of clustering. Accounting for two interim analyses utilizing a Haybittle-Peto stopping guideline set at $P<0.001$, for the study to have $80 \%$ power with a two-tailed type I error of 0.05 , a sample size of 230 infants was required.

All analyses were performed on an intention to treat basis. Continuous data are expressed as means with standard deviations or as medians with interquartile ranges (IQR), whereas categorical variables are expressed with frequencies and proportions. The primary outcome and other binary outcomes occurring in at least $5 \%$ of patients were analyzed using Pearson chi-squared tests. Less common binary outcomes were compared using Fisher's exact tests. Risk differences were calculated, along with their $95 \%$ confidence intervals.

Normally distributed continuous outcomes were compared using the Student's $t$ test, whereas Wilcoxon ranksum tests were used to compare continuous outcomes with skewed distributions. Planned analyses were performed to assess treatment effect heterogeneity across several clinical characteristics known to be associated with CPAP treatment failure: gestational age, birth weight, and exposure to antenatal corticosteroids [22]. Treatment effect heterogeneity was tested by evaluating the significance of interactions between the factors of interest and treatment arm in $\log$ binomial regression models that included the factor, treatment arm, and their interactions. Regression models were estimated using generalized estimating equations, to account for the inherent correlations expected with multiples.

In a sensitivity analysis, in order to account for the correlation in outcomes expected within multiples and the stratification of randomization by gestational age, we fit marginal regression models. Robust standard errors were estimated, and all models included gestational age $\left(22^{0 / 7}\right.$ $<27$ weeks' gestation; 27-296/7 weeks' gestation). Log binomial regression models were fit for dichotomous outcomes, negative binomial regression models for count outcomes, linear regression models for continuous outcomes, Cox proportional hazards models for time to event outcomes, and cumulative logistic regression models for ordinal outcomes. In addition, we performed sensitivity analyses following the exclusion of either: 1) infants with a delayed (after randomization) diagnosis of major congenital anomaly; or 2) infants with protocol deviations, whether the deviation occurred before or after primary outcome determination. Analyses were performed using Statistical Analysis Software (SAS) Enterprise Guide version 7.15 (SAS Institute Inc., Cary, NC, USA).

\section{Data and safety monitoring committee}

Prior to trial commencement, a DSMC was appointed and consisted of four health care providers with expertize in neonatology, resuscitation, and clinical trial statistics. A priori stopping rules for adverse events and efficacy were established. Interim analyses were conducted by the DSMB following completion of study of 25 and 110 infants, respectively. The analyses compared the two groups with respect to efficacy, safety, and futility. The interim analyses were completed in June 2017 and January 2018. Based on these analyses, the DSMB recommended that the trial continue without modification. 


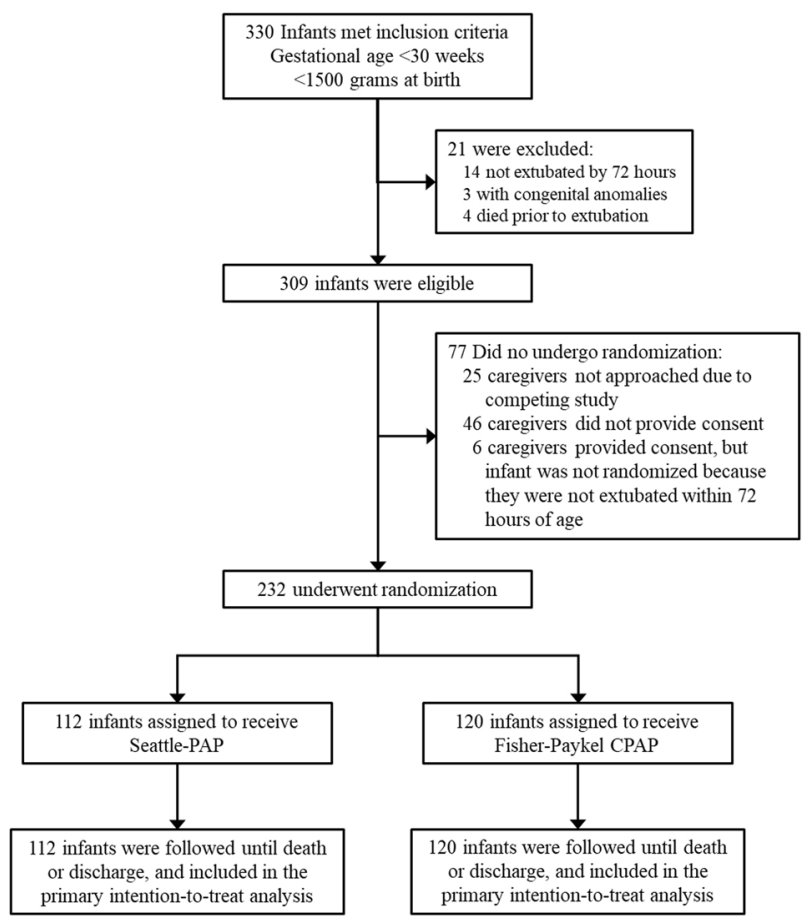

Fig. 2 Screening, randomization, and study completion. From March 24th, 2017 to January 5th, 2019, a total of five NICUs screened 330 infants who met the inclusion criteria, of whom 309 were eligible and 21 were excluded. The caregivers of 25 infants were not approached for informed consent, the caregivers of 46 did not provide consent, and six caregivers provided consent, but the infant did not undergo randomization after failing to extubate within $72 \mathrm{~h}$ of life. A total of 232 infants were randomized and enrolled. CPAP denotes continuous positive airway pressure.

\section{Results}

\section{Study population}

The trial took place from March 24th, 2017 to January 5th, 2019. A total of 232 premature infants underwent randomization (112 to the Seattle-PAP group and 120 to the FP-CPAP group, (Fig. 2). Infants in the Seattle-PAP and FP-CPAP groups had mean gestational ages (GA) of 27.0 and 27.2 weeks, respectively. Among eligible infants whose parents/guardians were approached for study participation and were extubated $<72 \mathrm{~h}$ of age, $83.5 \%$ (232/278) were consented and randomized. Baseline maternal and infant characteristics (Table 1) were well balanced between the groups, although the proportions of male infants and mothers selfidentified as black or African American were higher in the Seattle-PAP group than in the FP-CPAP group.

\section{Primary outcome}

Rates of CPAP treatment failure did not differ between Seattle-PAP (40/112, 35.7\%) and FP-CPAP (38/120,
Table 1 Study population characteristics.

\begin{tabular}{|c|c|c|}
\hline Maternal characteristics & Seattle-PAP $(n=103)$ & FP-CPAP $(n=105)$ \\
\hline Primigravida—no. (\%) & $34(33.0)$ & $40(38.1)$ \\
\hline Age-years & $28.8 \pm 6.2$ & $28.3 \pm 6.0$ \\
\hline \multicolumn{3}{|l|}{ Race or ethnicity ${ }^{\mathrm{a}}$-no. (\%) } \\
\hline Caucasian & $71(68.9)$ & $78(74.3)$ \\
\hline Black or African American & $28(27.2)$ & $16(15.2)$ \\
\hline Hispanic & $0(0)$ & $5(4.8)$ \\
\hline Asian or Pacific Islander & $2(1.9)$ & $0(0)$ \\
\hline Multiracial & $9(8.7)$ & $5(4.8)$ \\
\hline Other & $2(1.9)$ & $2(1.9)$ \\
\hline Unknown & $0(0)$ & $1(1.0)$ \\
\hline \multicolumn{3}{|l|}{ Maternal marital status-no. $(\%)^{\mathrm{b}}$} \\
\hline Single & $50(48.5)$ & $53(50.5)$ \\
\hline Married & $53(51.5)$ & $50(47.6)$ \\
\hline Mother in pre-term labor-no. (\%) & $62(60.2)$ & $63(60.0)$ \\
\hline Rupture of membranes—no. (\%) & $38(36.9)$ & $44(41.9)$ \\
\hline Period prior to delivery (hours) ${ }^{c}$ & $120(0-1224)$ & $71(0-1056)$ \\
\hline Diagnosis of chorioamnionitis - no. $(\%)$ & $25(24.3)$ & $25(23.8)$ \\
\hline Antenatal glucocorticoids received-no. (\%) & $93(90.3)$ & $101(96.2)$ \\
\hline Number of doses of antenatal corticosteroids & $2(1-4)$ & $2(1-4)$ \\
\hline \multicolumn{3}{|l|}{ Delivery method-no. (\% of infants) } \\
\hline Vaginal & $32(28.6)$ & $42(35.0)$ \\
\hline Cesarean section & $80(71.4)$ & $78(65.0)$ \\
\hline Infant Characteristics & $\begin{array}{l}\text { Seattle-PAP } \\
(n=112)\end{array}$ & $\begin{array}{l}\text { FP-CPAP } \\
(n=120)\end{array}$ \\
\hline Gestational age (weeks) & $27.0 \pm 1.8$ & $27.2 \pm 1.7$ \\
\hline$<27$ wk-no. $(\%)$ & $36(32.1)$ & $39(32.5)$ \\
\hline $27-<30$ wk-no. $(\%)$ & $76(67.9)$ & $81(67.5)$ \\
\hline Birth weight (grams) & $990 \pm 253$ & $1020 \pm 254$ \\
\hline Male sex $(\%)-$ no. $(\%)$ & $70(62.5)$ & $59(49.2)$ \\
\hline Multiple gestation $(\%)-$ no. $(\%)$ & $25(22.3)$ & $34(28.3)$ \\
\hline $\begin{array}{l}\text { Intubated in the delivery room- } \\
\text { no. }(\%)\end{array}$ & $49(43.8)$ & $44(36.7)$ \\
\hline \multicolumn{3}{|l|}{ Surfactant treatment-no. (\%) } \\
\hline In the delivery room & $31(27.7)$ & $38(31.7)$ \\
\hline At any time & $74(66.1)$ & $73(60.8)$ \\
\hline $\begin{array}{l}\text { Apgar scores at five minutes, } \\
\text { median (IQR) }^{\mathrm{e}}\end{array}$ & $7(5-8)$ & $7(6-8)$ \\
\hline $\begin{array}{l}\text { Postnatal age at randomization, } \\
\text { median (IQR)—-hours }\end{array}$ & $8(0-17.8)$ & $10(0-20.0)$ \\
\hline $\begin{array}{l}\text { Fraction of inspired oxygen at } \\
\text { randomization, median (IQR) }\end{array}$ & $25.0(21-30)$ & $24.5(21-30)$ \\
\hline $\begin{array}{l}\text { Age at randomization, median } \\
\text { (range)-hours }\end{array}$ & $8(0-60)$ & $10(0-70)$ \\
\hline $\begin{array}{l}\text { Caffeine received in first } 24 \mathrm{~h} \text { of } \\
\text { life-no. }(\%)\end{array}$ & $111(99.1)$ & $119(99.2)$ \\
\hline
\end{tabular}

No significant differences between the treatment groups for any characteristics were observed.

Values reported are means \pm SD or Median (Interquartile Range IQR). All other variables are reported as frequencies and percentages.

${ }^{\mathrm{a}}$ Race and ethnicity were self-reported.

${ }^{b}$ Not indicated in two cases from FP-CPAP group.

${ }^{\mathrm{c}}$ Duration of rupture of membranes was not reported in 12 cases.

${ }^{\mathrm{d}}$ Clinical diagnosis of chorioamnionitis.

${ }^{\mathrm{e}} \mathrm{APGAR}$ scores at $5 \mathrm{~min}$ not reported $(n=2)$. 
31.7\%; risk difference, 4.1 percentage points; $95 \% \mathrm{CI}$, $-8.1-16.2 ; P=0.51)$. Treatment effect heterogeneity by gestational age, birth weight, or exposure to antenatal corticosteroids was not observed (Table 2).

\section{Secondary outcomes}

The most common reason for treatment failure was respiratory distress requiring intubation (Table 3), with no between-group differences. Treatment failures were most likely to occur during the first hours after randomization (Supplemental Fig. 1). After day 2, fewer than half of the infants in both groups were undergoing ventilation. Among mother-infant dyads consented antenatally and started on CPAP at birth, we observed no differences between groups in rates of treatment failures (Seattle-PAP: 4/32, 12.5\%; FPCPAP: $10 / 35,35.6 \% ; P=0.14)$. The duration of hospitalization was similar among infants in the Seattle-PAP (median 82 days, IQR 39-260) and FP-CPAP (median 82, IQR 37-285; $P=0.71)$ groups.

\section{Adverse events, adherence to respiratory guidelines, and protocol violations}

We observed no differences between the groups in the frequencies of pre-specified adverse events (Supplementary Table 1). No deaths or adverse events were attributable to either device. The causes of death included sepsis associated with disseminated intravascular coagulation $(n=5)$, necrotizing enterocolitis (NEC) refractory to medical/surgical care $(n=2)$, pulmonary hemorrhage $(n=2)$, respiratory failure $(n=2)$, withdrawal of support due to severe intraventricular hemorrhage $(n=1)$, and spontaneous intestinal perforation $(n=1)$. Adherence to respiratory guidelines was high; five cases of nonadherence centered around the use of nasal cannula, rather than CPAP, among infants requiring $\mathrm{FiO}_{2}>0.21$ prior to 32 weeks' gestational age. Study protocol violations were typically administrative errors that resulted in the use of FP-CPAP in 7.1\% (8/112) of infants assigned to Seattle-PAP and in the use of Seattle-PAP in $0.8 \%(1 / 120)$ of infants assigned to FP-CPAP. These violations were quickly corrected $(<6 \mathrm{~h})$ to limit exposure. Additional violations included transfer to a non-study site $(n=3)$ or physician request for FP-CPAP rather than assigned Seattle-PAP device $(n=1)$.

\section{Sensitivity analysis}

We observed no differences in primary or secondary outcomes following the exclusion of infants $(n=5)$ with a delayed diagnosis of a major cardiac or lung malformation. In addition, we observed no differences in primary or
Table 2 Characteristics of patients who experienced treatment failure.

\begin{tabular}{llll}
\hline Characteristics & $\begin{array}{l}\text { Seattle-PAP } \\
(n=112)\end{array}$ & $\begin{array}{l}\text { FP-CPAP } \\
(n=120)\end{array}$ & $P$ Value \\
\hline $\begin{array}{l}\text { Gestational age } \\
\quad<27 \text { weeks } \\
\quad(n=36,39)\end{array}$ & $26(72.2)$ & $31(79.5)$ & 0.06 \\
$\quad \begin{array}{l}27-<30 \text { weeks } \\
(n=76,81)\end{array}$ & $14(18.4)$ & $7(8.6)$ & \\
$\begin{array}{l}\text { Birth weight } \\
\quad<750 \mathrm{~g}(n=18,17)\end{array}$ & $14(77.8)$ & $16(94.1)$ & \\
$\quad \begin{array}{l}750-1499 \mathrm{~g} \\
(n=94,103)\end{array}$ & $26(27.7)$ & $22(21.4)$ & \\
$\begin{array}{l}\text { Antenatal corticosteroids } \\
\text { received }\end{array}$ & & & \\
$\quad \begin{array}{l}\text { No }(n=11,3) \\
\text { Yes }(n=101,116)\end{array}$ & $34(33.7)$ & $35(30.2)$ & \\
\hline
\end{tabular}

$P$ values are from tests of heterogeneity of the treatment effect on the relative risk scale, estimated using log binomial regression models fit using generalized estimating equations. The number $(N)$ of infants within each subgroup are noted in parentheses in the first column. Incidence data for subgroup comparisons are shown as $N(\%)$.

secondary outcomes following the exclusion of infants $(n=13)$ with protocol deviations.

\section{Discussion}

Preclinical studies showed that Seattle-PAP provides more variable pressure oscillations, along with a broader range of frequencies and with a shift to lower frequencies of pressure oscillations, than traditional bubble CPAP [11, 23]. These properties suggested that Seattle PAP would improve air exchange and better address the stochastic properties of newborn infants' lungs [8]. Initial clinical studies among larger, more mature infants with lower supplemental oxygen requirements than infants in the present study, reported that the effort of breathing was lower in the infants supported with Seattle-PAP than with traditional bubble CPAP [12]. The lack of beneficial effects of Seattle PAP in the population in the present studies suggests a number of possible interpretations.

In premature infants, respiratory failure is a complex disorder, variously attributed to structurally and functionally immature lungs, compliant chest walls, obstructive and/or central apnea, alone or in combination $[8,24,25]$. While the primary outcome (CPAP failure) is consistent with previous studies $[18,26]$, we acknowledge the need for intubation and mechanical ventilation (CPAP failure) may not reflect primary respiratory failure. To that end, CPAP failure can reflect a variety of etiologies, including airway obstruction, nasal obstruction, and gastric distention [20]. In contrast to adult respiratory failure, identifiable phenotypes for 
Table 3 Reasons for treatment failure and secondary outcomes.

\begin{tabular}{|c|c|c|c|c|}
\hline Outcome & $\begin{array}{l}\text { Seattle-PAP } \\
(n=112)\end{array}$ & $\begin{array}{l}\text { FP-CPAP } \\
(n=120)\end{array}$ & $\begin{array}{l}\text { Risk Difference } \\
(95 \% \mathrm{CI})\end{array}$ & $P$ value \\
\hline \multicolumn{5}{|l|}{ Reason for treatment failure ${ }^{a}$} \\
\hline Failure of successful transition ${ }^{\mathrm{b}}$ & $15(13.4)$ & $18(15.0)$ & $-1.6(-10.6-7.4)$ & 0.73 \\
\hline Increase in fraction of inspired oxygen & $15(13.4)$ & $22(18.3)$ & $-4.9(-14.3-4.4)$ & 0.30 \\
\hline Escalation of respiratory care requiring intubation ${ }^{c}$ & $22(19.6)$ & $24(20.0)$ & $-0.4(-10.6-9.9)$ & 0.95 \\
\hline Escalation of respiratory care to $\mathrm{SiPAP}$ & $3(2.7)$ & $0(0)$ & $2.7(-0.3-5.7)$ & 0.11 \\
\hline $\begin{array}{l}\text { Number of days on mechanical ventilation after trial entry, } \\
\text { median (IQR) })^{\mathrm{d}}\end{array}$ & $0(0-4)$ & $0(0-5)$ & NA & 0.20 \\
\hline $\begin{array}{l}\text { Number of days any positive pressure support after trial entry, } \\
\text { median (IQR) })^{\text {e,f }}\end{array}$ & $47.5(34.5-75.5)$ & $51(32-74)$ & NA & 0.99 \\
\hline $\begin{array}{l}\text { Number of days of oxygen therapy after trial entry, median } \\
(\mathrm{IQR})^{\mathrm{f}}\end{array}$ & $28.5(5-91)$ & $34(5-84)$ & NA & 0.63 \\
\hline Number of days on room air after trial entry, median $(\mathrm{IQR})^{\mathrm{f}}$ & $46(19-57)$ & $43(20-60)$ & NA & 0.93 \\
\hline $\begin{array}{l}\text { Number of days to achieve full enteral feeding after trial } \\
\text { entry, median }(\mathrm{IQR})^{\mathrm{f}, \mathrm{g}}\end{array}$ & $12.5(10-14)$ & $11(10-14)$ & NA & 0.73 \\
\hline Number of days to achieve full-suck feeding, median (IQR) ${ }^{\mathrm{f}}$ & $73(63-80)$ & $78(66-83)$ & NA & 0.95 \\
\hline Weight at discharge $(\mathrm{kg})^{\mathrm{f}}$ & $3.1(2.6-3.8)$ & $3.0(2.6-4.1)$ & NA & 0.89 \\
\hline
\end{tabular}

Definition of room air includes infants who were on CPAP or nasal cannula with FiO2 of 0.21 .

SiPAP synchronized inspiratory positive airway pressure, IQR Interquartile range, NA Not applicable.

${ }^{\text {a }}$ Treatment may have failed for more than one reason.

${ }^{\mathrm{b}}$ Intubation within $72 \mathrm{~h}$ for surfactant administration after initiation of bn-CPAP and then fails to meet extubation criteria by $72 \mathrm{~h}$.

${ }^{c}$ Intubation was recommended if more than two episodes of apnea requiring bag-mask ventilation were encountered or more than six episodes of apnea required stimulation in a $24 \mathrm{~h}$ period.

${ }^{\mathrm{d}}$ Mechanical ventilation includes synchronized intermittent ventilation (SIMV) and high-frequency oscillatory ventilation (HFOV).

${ }^{\text {e }}$ Respiratory support included the use of nasal continuous positive airway pressure, SIMV, and HFOV.

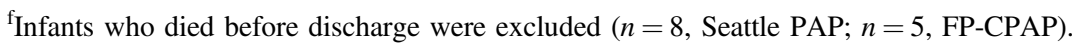

${ }^{\mathrm{g}}$ Full enteral and full-suck feeding defined as feeds $>130 \mathrm{~mL} / \mathrm{kg} /$ day.

respiratory failure, based on clinical criteria or biomarkers, are not readily available in preterm infants [27]. While no evidence of heterogeneity of treatment effect across the reasons for treatment failure were observed, the study was not powered to detect potential differences among subgroups. Further studies may identify subgroups of infants who might have greater responses to Seattle-PAP than were observed in our study, such as the earlier studies on SeattlePAP that involved larger, more mature infants with respiratory failure largely attributable to primary respiratory dysfunction $[8,12]$. In following the common practice of alternating the interface used to deliver CPAP between nasal prongs and mask, designed to minimize nasal injury, inconsistent delivery of distending pressures may have dampened the Seattle-PAP pressure fluctuations and obscured any differences between the two groups $[9,12]$.

The strengths of this study include participation by a network of five NICU sites that share a consistent approach to the care of premature infants [14]. Rates of parental/ caregiver consent were high $(>80 \%)$ and reflect a motivated group of health care providers and families. The variety of included sites (size, location, and academic/private) increases the generalizability of findings. Absence of any observable differences in rates of pneumothorax, NEC, or other adverse effects, associated with use of Seattle-PAP is notable. Without clear advantages of either therapy, health care providers may choose the device that is associated with less resource utilization or greater convenience [28]. To inform decisions about resource allocation, a formal economic evaluation is ongoing [29].

In part, Seattle-PAP was designed and developed to address high rates of CPAP failure among low-and middleincome countries (LMIC) $[8,12]$. The design and context of the present study has limited applicability to LMIC settings; thus, observed findings should not preclude the execution and conduct of a large, RCT of Seattle-PAP in LMIC. In fact, consistent with the calls from previous investigators, such trials are critically necessary to better characterize the skills, organization, and resources necessary to optimize bubble CPAP in LMIC [30-32].

Our study has several limitations. The current study used a superiority design; thus, findings cannot be interpreted to show noninferiority of Seattle-PAP to FP-CPAP. Since the study was conducted at a single academic center, the primary 
outcome was short-term treatment efficacy (CPAP treatment failure), rather than death or morbidity (BPD). Although our interventions did not permit blinding of treatment assignment to health care providers, thresholds for respiratory failure were based on pre-specified, objective criteria to minimize bias [13]. Although care might have been affected by knowledge of treatment allocation, outcome assessors were not privy to group allocation. Meticulous attention to optimize device positioning in the current study likely contributed to low rates of device-related complications (e.g., nasal injury), but the requisite skills to optimize bubble CPAP may not be routine in many health care systems.

Regardless of treatment assignment, CPAP failure rates were high among infants born at $<27$ weeks, particularly those born at $\leq 24$ weeks of gestation, and additional therapeutic strategies are needed [9]. Previous well-designed, large RCTs evaluating CPAP as a primary mode of respiratory support reported rates of CPAP failure of 46 and $51 \%$, respectively $[5,6]$. While rates of CPAP failure in the present study are lower than those of previous trials, differences in clinical characteristics (gestational age, use of surfactant) and definitions for CPAP failure (timing, thresholds) preclude meaningful comparisons [5, 6]. Markedly variable CPAP failure rates among centers reflect a number of determinants, including time and experience with CPAP, and the level and extent of multidisciplinary collaboration [9, 33]. Previous investigators have characterized pragamatic, interdisciplinary strategies to reduce CPAP failure rates, with the ultimate goal of reducing rates of chronic lung disease and improving neonatal outcomes [20].

Even though more than 230 preterm infants were enrolled, rates of many primary outcomes were low, limiting our statistical power to detect differences between groups. Although patients were only eligible to enter the study if they were admitted to one of the participating NICUs within $72 \mathrm{~h}$ of birth, adjustment could not be made for care provided prior to transfer, which is a possible confounder in the analysis.

In conclusion, among premature infants born at $<30$ weeks of gestation, we observed no differences between Seattle-PAP and FP-CPAP in rates of CPAP treatment failure, mortality, morbidity, or the duration of hospitalization. The absence of any adverse events associated with Seattle-PAP is notable, wherein potential differences in resource utilization between the two devices must be considered. Additional studies are needed to determine whether Seattle-PAP could prove to be useful in offering practical respiratory support in resource-limited health care settings.

\section{Data availability}

The data will be made available from the global datasharing enterprise Vivli (https://vivli.org/resources). The
Vivli platform provides an independent data repository, an in-depth search engine, and a secure cloud-based analytics platform.

Acknowledgements The authors wish to acknowledge the doctors, nurses, respiratory therapists, and other hospital staff in the cooperating hospitals for their efforts. The authors wish to acknowledge Edward Shepherd MD, Thomas Bartman MD, and the Cure-Me BPD team at Nationwide Children's Hospital, who designed and implemented the respiratory treatment algorithm. The authors also wish to acknowledge the members of the data and safety monitoring committee (DSMB): Kevin C. Dysart, MD (chair), Children's Hospital of Philadelphia, Philadelphia PA; Hany Z. Aly, MD, Cleveland Clinic Children's Hospital, Cleveland, OH; Patricia R. Chess MD, Golisano Children's Hospital University of Rochester. Rochester, NY; Gary R. Cutter, PhD, University of Alabama at Birmingham, Birmingham, AL. Finally, the authors wish to acknowledge Edward Shepherd MD, Thomas Bartman MD, and the Cure-Me BPD team at $\mathrm{NCH}$, who designed and implemented the respiratory treatment algorithm.

Funding The study sponsor was the Seattle Children's Research Institute (Seattle, WA), with funding provided by the Bill \& Melinda Gates Foundation (grant number OPP1094124 to TNH, CVS). Neither of these entities played a role in the design of the study, collection, analysis, and interpretation of the data, preparation of the paper, or the decision to submit the paper for publication.

\section{Compliance with ethical standards}

Conflict of interest CPR, TNH, and CVS are included among the holders of patents on the Seattle-PAP device, specifically United States Patent \#8499759, under the title "Broad-band, low frequency, highamplitude, long time duration, oscillating airway pressure breathing apparatus and method utilizing bubbles". The patent is also registered with the World Intellectual Property Organization (PCT/US2009/ 039957), with patents or letters granted in several regions or countries, including Europe (2303373), Korea (10-1,540,948), New Zealand (588682), China (201310269076.X), Canada (2720976), Vietnam (13756), and India (7898/DELNP/2010). A patent has also been issued in Australia (2010241205) under a modified name. The rights to the Seattle-PAP device have been licensed to Draeger, Inc. CHB is a consultant for Abbott. The remaining authors have no other interests to declare.

Publisher's note Springer Nature remains neutral with regard to jurisdictional claims in published maps and institutional affiliations.

Open Access This article is licensed under a Creative Commons Attribution 4.0 International License, which permits use, sharing, adaptation, distribution and reproduction in any medium or format, as long as you give appropriate credit to the original author(s) and the source, provide a link to the Creative Commons license, and indicate if changes were made. The images or other third party material in this article are included in the article's Creative Commons license, unless indicated otherwise in a credit line to the material. If material is not included in the article's Creative Commons license and your intended use is not permitted by statutory regulation or exceeds the permitted use, you will need to obtain permission directly from the copyright holder. To view a copy of this license, visit http://creativecommons. org/licenses/by/4.0/. 


\section{References}

1. Kirpalani H, Millar D, Lemyre B, Yoder BA, Chiu A, Roberts RS, et al. A trial comparing noninvasive ventilation strategies in preterm infants. N Engl J Med. 2013;369:611-20.

2. American Academy of Pediatrics Committee on Fetus and Newborn. Respiratory support in preterm infants at birth. Pediatrics. 2014;133:171-4.

3. Wright CJ, Polin RA, Kirpalani H. Continuous positive airway pressure to prevent neonatal lung injury: How did we get here, and how do we improve? J Pediatr. 2016;173:17-24. e12.

4. Dunn MS, Kaempf J, de Klerk A, de Klerk R, Reilly M, Howard $\mathrm{D}$, et al. Randomized trial comparing 3 approaches to the initial respiratory management of preterm neonates. Pediatrics. 2011; 128:e1069-76.

5. Morley CJ, Davis PG, Doyle LW, Brion LP, Hascoet JM, Carlin $\mathrm{JB}$, et al. Nasal CPAP or intubation at birth for very preterm infants. N Engl J Med. 2008;358:700-8.

6. SUPPORT Study Group of the Eunice Kennedy Shriver Neonatal Research Network, Finer NN, Carlo WA, Walsh MC, Rich W, Gantz MG, et al. Early CPAP versus surfactant in extremely preterm infants. N Engl J Med. 2010;362:1970-9.

7. Sandri F, Plavka R, Ancora G, Simeoni U, Stranak Z, Martinelli S, et al. Prophylactic or early selective surfactant combined with nCPAP in very preterm infants. Pediatrics. 2010;125:e1402-09.

8. Welty SE. Continuous positive airway pressure strategies with bubble nasal continuous positive airway pressure: Not all bubbling is the same: The Seattle positive airway pressure system. Clin Perinatol. 2016;43:661-71.

9. Wright CJ, Sherlock LG, Sahni R, Polin RA. Preventing continuous positive airway pressure failure: Evidence-based and physiologically sound practices from delivery room to the neonatal intensive care unit. Clin Perinatol. 2018;45:257-71.

10. Diblasi RM. Nasal continuous positive airway pressure (CPAP) for the respiratory care of the newborn infant. Respir Care. 2009;54:1209-35.

11. Diblasi RM, Zignego JC, Tang DM, Hildebrandt J, Smith CV, Hansen TN, et al. Noninvasive respiratory support of juvenile rabbits by high-amplitude bubble continuous positive airway pressure. Pediatr Res. 2010;67:624-9.

12. Welty SE, Rusin CG, Stanberry LI, Mandy GT, Gest AL, Ford JM, et al. Short term evaluation of respiratory effort by premature infants supported with bubble nasal continuous airway pressure using Seattle-PAP and a standard bubble device. PLoS ONE. 2018;13:e0193807. https://doi.org/10.1371/journal.pone.0193807.

13. Backes CH, Notestine JL, Lamp JM, Balough JC, Notestine AM, Alfred CM, et al. Evaluating the efficacy of Seattle-PAP for the respiratory support of premature neonates: study protocol for a randomized controlled trial. Trials. 2019;20:63. https://doi.org/10. 1186/s13063-018-3166-6.

14. Nankervis CA, Martin EM, Crane ML, Samson KS, Welty SE, Nelin LD. Implementation of a multidisciplinary guideline-driven approach to the care of the extremely premature infant improved hospital outcomes. Acta Paediatr. 2010;99:188-93.

15. American Academy of Pediatrics and American Heart Association, Editors. Neonatal Resuscitation Textbook. 6th Edition. Elk Grove: American Academy of Pediatrics; 2011.

16. Perlman JM, Wyllie J, Kattwinkel J, Wyckoff MH, Aziz K, Guinsburg R, et al. Part 7: Neonatal Resuscitation: 2015 International consensus on cardiopulmonary resuscitation and emergency cardiovascular care science with treatment recommendations. Circulation. 2015;132(16 Suppl 1):S204-41.

17. Kirpalani H, Ratcliffe SJ, Keszler M, Davis PG, Foglia EE, Te Pas A, et al. Effect of sustained inflations vs intermittent positive pressure ventilation on bronchopulmonary dysplasia or death among extremely preterm infants: The SAIL randomized clinical trial. JAMA. 2019;321:1165-75.

18. Roberts CT, Owen LS, Manley BJ, Froisland DH, Donath SM, Dalziel KM, et al. Nasal high-flow therapy for primary respiratory support in preterm infants. N Engl J Med. 2016;375:1142-51.

19. Polin RA, Sahni R. Newer experience with CPAP. Semin Neonatol. 2002;7:379-89.

20. Sahni R, Schiaratura M, Polin RA. Strategies for the prevention of continuous positive airway pressure failure. Semin Fetal Neonatal Med. 2016;21:196-203.

21. Newnam KM, McGrath JM, Salyer J, Estes T, Jallo N, Bass WT. A comparative effectiveness study of continuous positive airway pressure-related skin breakdown when using different nasal interfaces in the extremely low birth weight neonate. Appl Nurs Res. 2015;28:36-41.

22. Dargaville PA, Gerber A, Johansson S, De Paoli AG, Kamlin CO, Orsini $\mathrm{F}$, et al. Incidence and outcome of CPAP failure in preterm infants. Pediatrics. 2016:138;e20153985.

23. Diblasi RM, Zignego JC, Smith CV, Hansen TN, Richardson CP. Effective gas exchange in paralyzed juvenile rabbits using simple, inexpensive respiratory support devices. Pediatr Res. 2010;68: 526-30.

24. Owen LS, Manley BJ, Davis PG, Doyle LW. The evolution of modern respiratory care for preterm infants. Lancet. 2017;389: 1649-59.

25. Reuter S, Moser C, Baack M. Respiratory distress in the newborn. Pediatr Rev. 2014;35:417-28.

26. Manley BJ, Owen LS, Doyle LW, Andersen CC, Cartwright DW, Pritchard MA, et al. High-flow nasal cannulae in very preterm infants after extubation. N. Engl J Med. 2013;369:1425-33.

27. Calfee CS, Delucchi K, Parsons PE, Thompson BT, Ware LB, Matthay MA, et al. Subphenotypes in acute respiratory distress syndrome: latent class analysis of data from two randomised controlled trials. Lancet Respir Med. 2014;2:611-20.

28. Zupancic JA, Dukhovny D. Resource distribution in neonatology: beyond the Pareto principle. Arch Dis Child Fetal Neonatal Ed. 2015;100:F472-3.

29. Huang L, Roberts CT, Manley BJ, Owen LS, Davis PG, Dalziel KM. Cost-effectiveness analysis of nasal continuous positive airway pressure versus nasal high flow therapy as primary support for infants born preterm. J Pediatrics. 2018;196:58-64. e52.

30. Lissauer T, Duke T, Mellor K, Molyneux L. Nasal CPAP for neonatal respiratory support in low and middle-income countries. Arch Dis Child Fetal Neonatal Ed. 2017;102:F194-6.

31. Martin S, Duke T, Davis P. Efficacy and safety of bubble CPAP in neonatal care in low and middle income countries: a systematic review. Arch Dis Child Fetal Neonatal Ed. 2014;99: F495-504.

32. McAdams RM. Bubble CPAP may be safe and efficacious for neonates in low and middle income countries, but more evidence is needed. Evid Based Med. 2015;20:62.

33. Aly H, Milner JD, Patel K, El-Mohandes AA. Does the experience with the use of nasal continuous positive airway pressure improve over time in extremely low birth weight infants? Pediatrics. 2004;114:697-702. 